\title{
Psoralen and Bergapten: In Silico Metabolism and Toxicophoric Analysis of Drugs Used to Treat Vitiligo
}

Vinicius Barreto da Silva'; Daniel Fábio Kawano'; Ivone Carvalho1; Edemilson Cardoso da Conceição; Osvaldo de Freitas $^{2}$; Carlos Henrique Tomich de Paula da Silva ${ }^{1}$

${ }^{1}$ Departamento de Ciências Farmacêuticas, Faculdade de Ciências Farmacêuticas de Ribeirão Preto, Universidade de São Paulo, Ribeirão Preto, Brazil

${ }^{2}$ Departamento de Tecnologia Farmacêutica, Faculdade de Farmácia, Universidade Federal de Goiás, Goiânia, Brazil

Received, July 31, 2009; Revised, September 22, 2009; Accepted, December 4, 2009; Published, December 5, 2009.

\begin{abstract}
Purpose. To discuss the contribution of psoralen and bergapten metabolites on psoralens toxicity. Methods. Computational chemistry prediction of metabolic reactions and toxicophoric groups based on the expert systems Derek and Meteor. Results. A total of 15 metabolites were suggested for both psoralen and bergapten based on phase 1 and 2 biotransformations until the 3rd generation. Five toxicophoric substructures were shared among psoralen, bergapten and their corresponding metabolites; one toxicophoric marker (resorcinol) was only identified in bergapten and its biotransformation products. Conclusion. Although the toxic effects of psoralens are well known and documented, there is little information concerning the role of their metabolites in this process. We believe this work adds to the knowledge of which molecular substructures are relevant to the process of metabolism and toxicity induction, thus, guiding the search and development of more effective and less toxic drugs to treat vitiligo.
\end{abstract}

\section{INTRODUCTION}

Vitiligo affects $1 \%$ of the world's population and this disease is characterized by loss of pigmentproducing cells on the skin, which results in irregular shaped spots that range in color from white to pink [1]. It is a disease of unknown etiology although several factors such as genetic, autoimmune and environmental components (e.g. stress and intense sunlight exposure) may contribute to its manifestation [2]. Current therapy aims to reestablish the integral functionality of the melanocytes through the use of topical corticosteroids, antioxidants and immunomodulators, UVB phototherapy and photochemotherapy using psoralens followed by UVA irradiation [3].

Psoralens (or linear furanocoumarins) are photosensibilising substances that enhance skin sensibility by enhancing melanin production. These compounds are widely distributed in the plant kingdom, being particularly abundant in Umbelliferae (Apiaceae) and Rutaceae [4]. These plants have been extensively used in the treatment of vitiligo since $1500 \mathrm{BC}$ [5] and psoralen and bergapten are the components that predominantly account for their therapeutic effects in those patients with vitiligo [6].

Psoralen and bergapten, as well as any compounds of the furanocoumarin class, exert their photosensibilising effects through a covalent interaction with DNA triggered by light of a specific wavelength $(320-410 \mathrm{~nm})$. The resulting complex blocks the DNA interaction with transcriptases and polymerases, avoiding cell replication $[7,8]$. This mechanism consist of 3 essential steps, i.e., (a) drug intercalation between DNA nucleotide bases, (b) drug absorption of a UVA photon and covalent bond formation between the furan ring double bond and a thymine base (T2) of the DNA molecule, (c) absorption of a second photon (UVA) and covalent bonding between the lactone ring double bond and another thymine base (T1), which, in the end, results in a psoralen cross-linked DNA [9] (Figure 1).

Corresponding Author: Vinicius Barreto da Silva, Departamento de Ciências Farmacêuticas, Faculdade de Ciências Farmacêuticas de Ribeirão Preto, Universidade de São Paulo, Avenida do Café S/N, Ribeirão Preto-SP, CEP 14040-903, Brasil. E-mail: vbs11@usp.br 


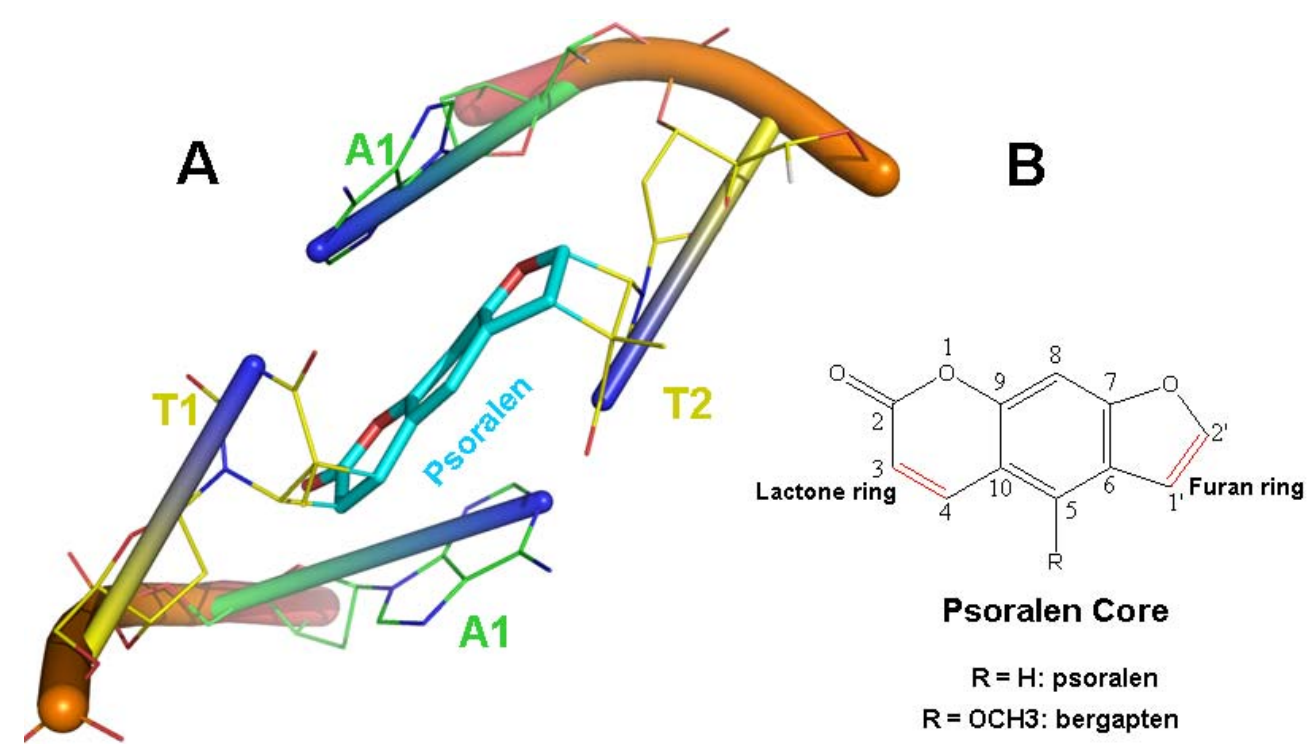

Figure 1. (A) Model for the cross-linkage between psoralen and thymine bases (T1 and T2). The psoralen molecule is intercalated between two pairs of the nucleotide bases adenine and thymine. (B) Chemical structure of psoralen core highlighting (in red) the sites in the furan and lactone ring which make covalent bonding with DNA molecule.

Although psoralen and bergapten can be useful in the therapy of vitiligo and other diseases, reports of adverse events associated with linear furanocoumarins are relatively common. Both teratogenicity [10] and carcinogenicity (mainly skin cancer) $[11,12]$ were related to the use of furanocoumarins but the most common clinical signs concerning the administration of psoralens is the photodermatitis, a reaction characterized by pruritic erythema, edema and vesiculation on the skin surface [13]. Nevertheless, few studies focusing on the influence of furanocoumarin metabolites on the toxicity of psoralens were published [14,15], and most of the current information regarding the metabolism of psoralen and bergapten is extrapolated from reported metabolism of classical coumarins.

Oral administration of coumarins in humans results in fast absorption through the gastrointestinal tract and metabolization via Cytochrome P450 2A6 to give the 7hydroxycoumarin, which is secreted into the urine as glucuronide and sulphate conjugates [16]. Although 7-hydroxycoumarin is the major metabolite, several secondary metabolites can be produced by hydroxylation at the 3 and 8 positions of psoralen core (Figure 1) or by lactone ring opening [17]. The hepatotoxicity of coumarins seems to be mediated by the metabolism in rodents, specifically through the generation of 3-hydroxylated metabolites and derivatives formed from heterocyclic ring rupture [18].

In line with these findings, additional studies to explore the relationship between the noxious effects of psoralens and their metabolism are necessary. In this work, we have used computational (in silico) chemistry methods in order to predict and investigate the generation of psoralen and bergapten metabolites and their possible relationship with the toxicity of these drugs (structure-toxicity relationship). We believe this work adds to the knowledge of which molecular substructures are relevant to the process of metabolism and toxicity induction, thus guiding the search and development of more effective and less toxic drugs.

\section{MATERIAL AND METHODS}

The software Meteor 10.0.2 [19] was used to predict the metabolic pathways of psoralen and bergapten. Simulations included phase I and II biotransformations processed until the $3^{\text {rd }}$ generation of metabolites, where all the molecules are conjugated or present low log $\mathrm{P}$ values and are more likely to be excreted. The analysis of toxicophoric groups regarding the structures of psoralen, bergapten and their proposed metabolites was carried out using the software Derek 10.0.2 [20] in order to model toxicity endpoints, including carcinogenicity, mutagenicity, genotoxicity, skin sensibilization, 
teratogenicity, airways hyperreactivity, hepatotoxicity, neurotoxicity, etc. Meteor and Derek identify metabolic reactions and toxicophoric groups (structural moieties that can exert toxic effects in a specific compound), respectively, through a knowledge-based system wherein structure-property correlations are searched from a database built from relevant literature concerning metabolic and toxicological data [21-24].

\section{RESULTS}

The biotransformation pathways proposed for psoralen and bergapten are shown in Tables 1 and 2, respectively. Table 1 describes the 15 psoralen predicted metabolites and Table 2 outlines the 15 bergapten predicted metabolites. All biotransformations involve the psoralen core and are generated by phase 1 and 2 metabolic reactions, according the results obtained during the 3 first generation analysis.

In Phase 1 reactions (oxidation, reduction, hydrolysis, hydroxylation) the structure of a xenobiotic molecule is modified, where a new functional group can be introduced, or an existing one can be modified or exposed. Phase 2 reactions are enzymatic synthesis where a functional group (alcohol or amine for example) is masked by the addition of a new group, such as glucuronic acid or sulfate. The objective of phase 1 and 2 biotransformations is to make the xenobiotic more polar and more easily extractable [25].

Figure 2 depicts the metabolites predicted from psoralen and bergapten structures. Based on the suggested metabolic pathway, the metabolites $\mathrm{P} 1$ and $\mathrm{B} 1$ can be produced by the hydrolysis of the coumarin ring, P4, B4 and P10 through epoxidation of the double bonds in the psoralen core and B10 via oxidative $O$-demethylation, which subsequently may generate $\mathrm{B} 11$ by glucuronidation at the $\mathrm{B} 8$ aromatic alcohol function.

Starting from $\mathrm{P} 1$ and $\mathrm{B} 1$, glucuronidation of the aromatic alcohol results respectively in $\mathrm{P} 2$ and $\mathrm{B} 2$. Conjugation of the unsaturation close to the carboxyl group with glutathione yields $\mathrm{P} 3$ and B3 and oxidative $O$-demethylation of B1 produces B12. B14 and B15 are formed by glucuronidation at distinct aromatic alcohols present in the aromatic ring of B12 and B13 is generated from a conjugation reaction with glutathione in the $\alpha, \beta$-unsaturation in B12 (Figure $3)$.

Five metabolites were predicted from $\mathrm{P} 4$ whereas the analysis of B4 resulted in six predicted metabolites (Figure 4). Specifically, oxidative $O$-demethylation of $\mathrm{B} 4$ produces only $\mathrm{B} 16$ and glutathione conjugation of $\mathrm{P} 4$ and $\mathrm{B} 4$ gave P8/B8 and P9/B9. Hydrolysis of P5 and B5 metabolites results in other four suggested metabolites (P6/B6 and P7/B7).

Regarding reactions comprising the metabolite P10, the epoxide group corresponds to the most susceptible group to metabolization via hydrolysis, glutathione conjugation and glucuronidation, yielding five new metabolites (Figure 5).

In silico toxicophoric analysis was performed of psoralen, bergapten and their metabolites. The results of this analysis and the corresponding endpoints of toxicity are outlined in Table $\mathbf{3}$ for psoralen and Table $\mathbf{4}$ for bergapten.

Table 1. Proposed biotransformation reactions starting from psoralen and its potential metabolites.

\begin{tabular}{ccl}
\hline Starting Molecule & Metabolite & Biotransformation \\
\hline Psoralen & P1 & Hydrolytic ring opening of coumarin \\
Psoralen & P4, P10 & Epoxidation \\
P1 & P2 & Glucuronidation of aromatic alcohol \\
P1 & P3 & Conjugation of $\alpha, \beta$-unsaturation with glutathione \\
P4 & P5 & Hydrolysis of vinyl ether epoxide \\
P4 & P8, P9 & Conjugation of vinyl ether epoxide with glutathione \\
P5 & P6, P7 & Hydrolysis of hemiacetal \\
P10 & P11, P12 & Conjugation of epoxide with glutathione \\
P10 & P13 & Vicinal diol from epoxide \\
P13 & P14, P15 & Glucuronidation of Alicyclic alcohol \\
\hline
\end{tabular}

Table 2. Proposed biotransformation reactions starting from bergapten and its potential metabolites. 


\begin{tabular}{ccl}
\hline Starting Molecule & Metabolite & Biotransformation \\
\hline Bergapten & B1 & Hydrolytic ring opening of coumarin \\
Bergapten & B4 & Epoxidation of furan \\
Bergapten & B10 & Oxidative O-demethylation \\
B1 & B2 & Glucuronidation of aromatic alcohol \\
B1 & B3 & Conjugation of $\alpha, \beta$-unsaturation with glutathione \\
B1 & B12 & Oxidative O-demethylation \\
B4 & B5 & Hydrolysis of vinyl ether epoxides \\
B4 & B8, B9 & Conjugation of epoxide with glutathione \\
B4 & B16 & Oxidative O-demethylation \\
B5 & B6, B7 & Hydrolysis of hemiacetal \\
B10 & B11 & Glucuronidation of aromatic alcohol \\
B12 & B13 & Conjugation of $\alpha, \beta$-unsaturation with glutathione \\
B12 & B14, B15 & Glucuronidation of aromatic alcohol \\
\hline
\end{tabular}

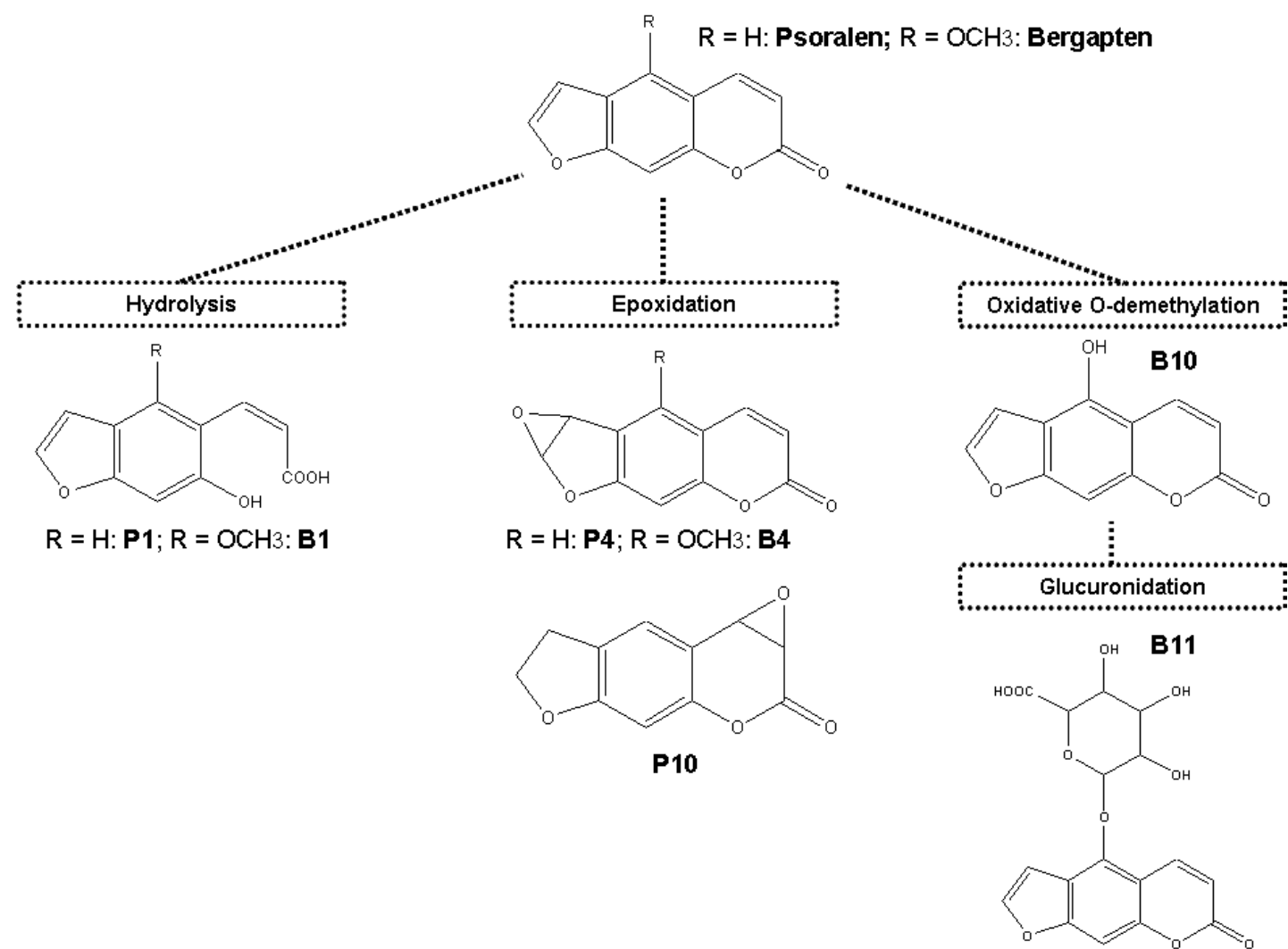

Figure 2. Metabolites and respective biotransformation reactions predicted for psoralen and bergapten using computational chemistry.

Five toxicophoric substructures were identified among psoralen, bergapten and their corresponding metabolites; specifically, psoralen, furan, epoxide, coumarin and phenyl ester. Resorcinol was only identified in bergapten and respective predicted biotransformation products (Table 4). The chemical structures of these toxicophoric groups are shown in Figure 6.

\section{DISCUSION}

In silico predictions of psoralen and bergapten biotransformations identified three potential first generation metabolites for each drug (Figure 2) produced by hydrolysis ( $\mathrm{P} 1$ and $\mathrm{B} 1$ ), epoxidation (P4, B4 and P10) and demethylation (B10). P4, $\mathrm{B} 4$ and P10 resulted from epoxidation at different positions of the furan and coumarin rings, 


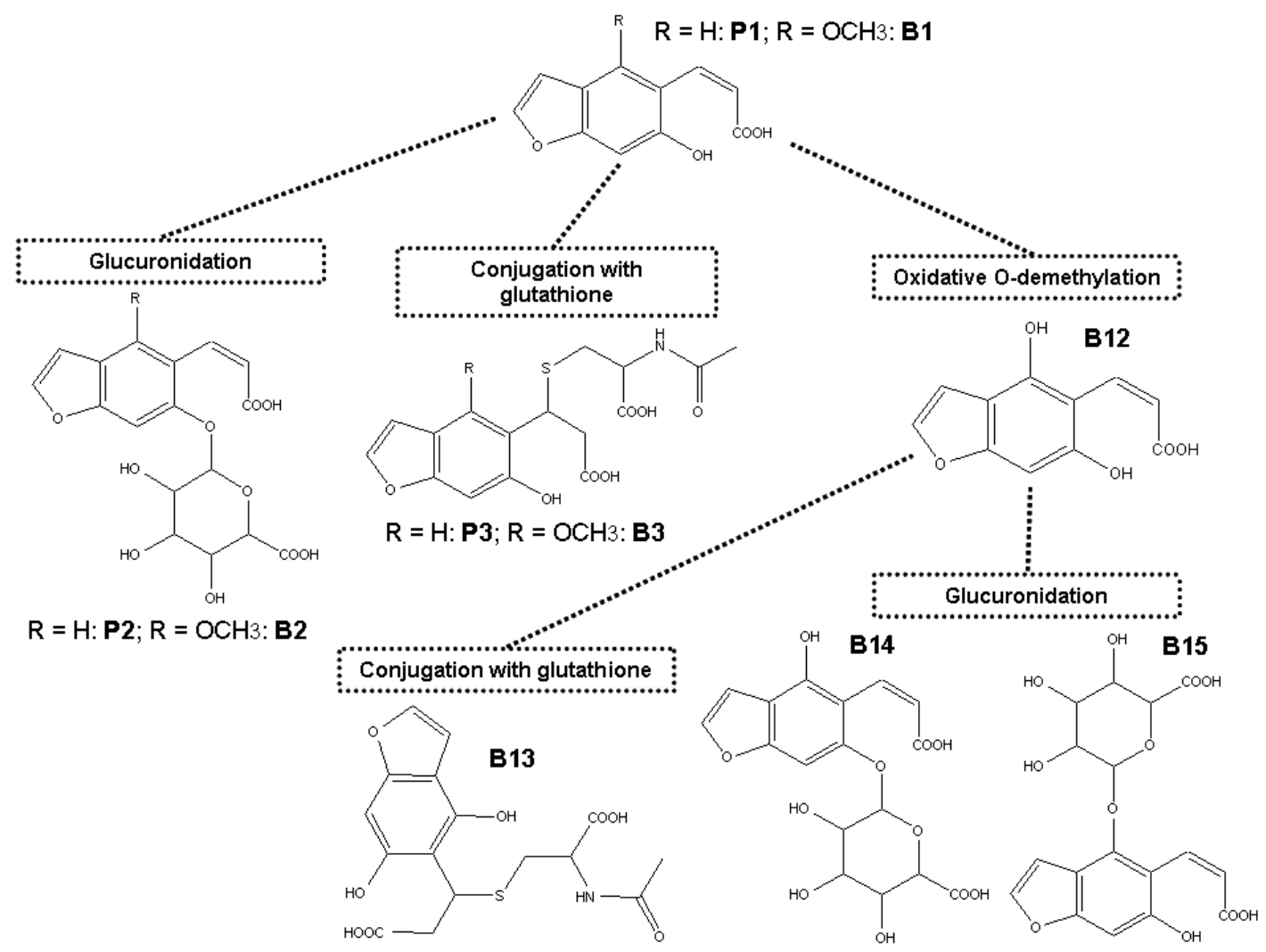

Figure 3. Metabolites and respective biotransformation reactions predicted for P1 and B1 using computational chemistry.

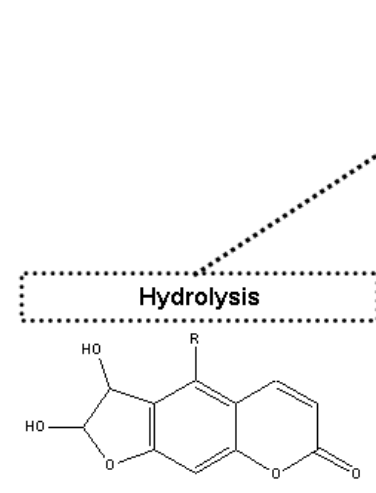

$\mathrm{R}=\mathrm{H}: \mathbf{P 5} ; \mathrm{R}=\mathrm{OCH} 3: \mathbf{B 5}$
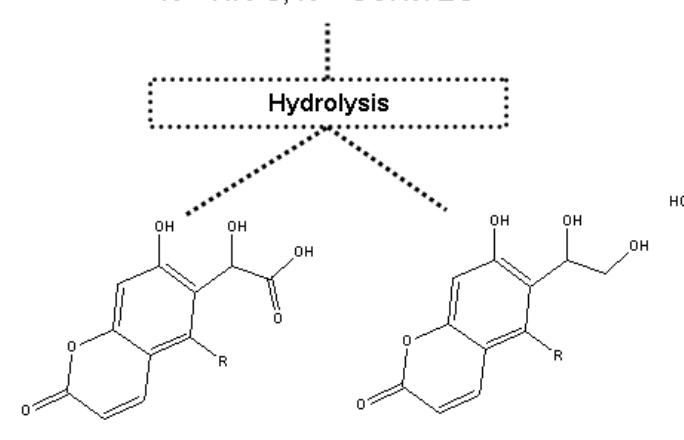

$\mathrm{R}=\mathrm{H}: \mathbf{P} 6 ; \mathrm{R}=\mathrm{OCH} 3: \mathbf{B} 6 \quad \mathrm{R}=\mathrm{H}: \mathbf{P} 7 ; \mathrm{R}=\mathrm{OCH}_{3}: \mathbf{B} 7$

$\mathrm{R}=\mathrm{H}: \mathbf{P 4} ; \mathrm{R}=\mathrm{OCH} 3: \mathbf{B 4}$

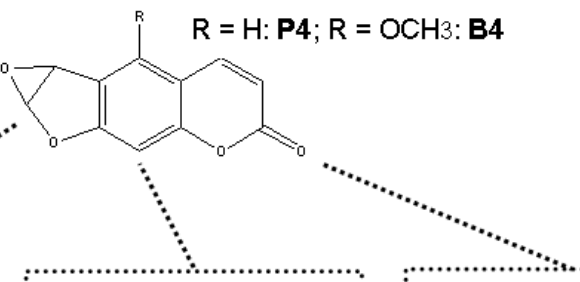

Oxidative O-demethylation $\quad$ Conjugation with glutathione

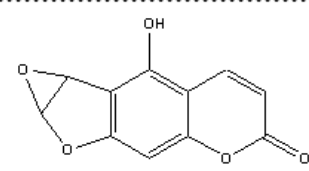

B16

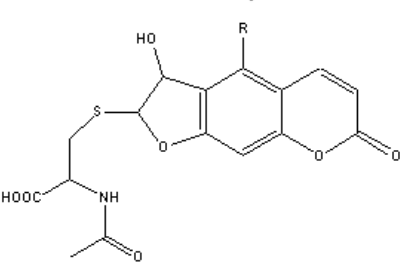

$\mathrm{R}=\mathrm{H}: \mathbf{P 8} ; \mathrm{R}=\mathrm{OCH} 3: \mathbf{B} 8$

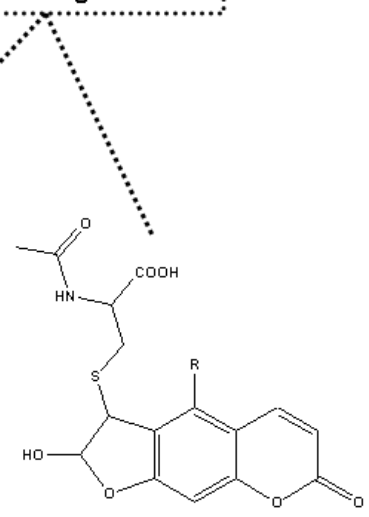

$\mathbf{R}=\mathrm{H}: \mathbf{P 9} ; \mathbf{R}=\mathrm{OCH} 3$ : $\mathbf{B 9}$

Figure 4. Metabolites and respective biotransformation reactions predicted for P4 and B4 using computational chemistry. 


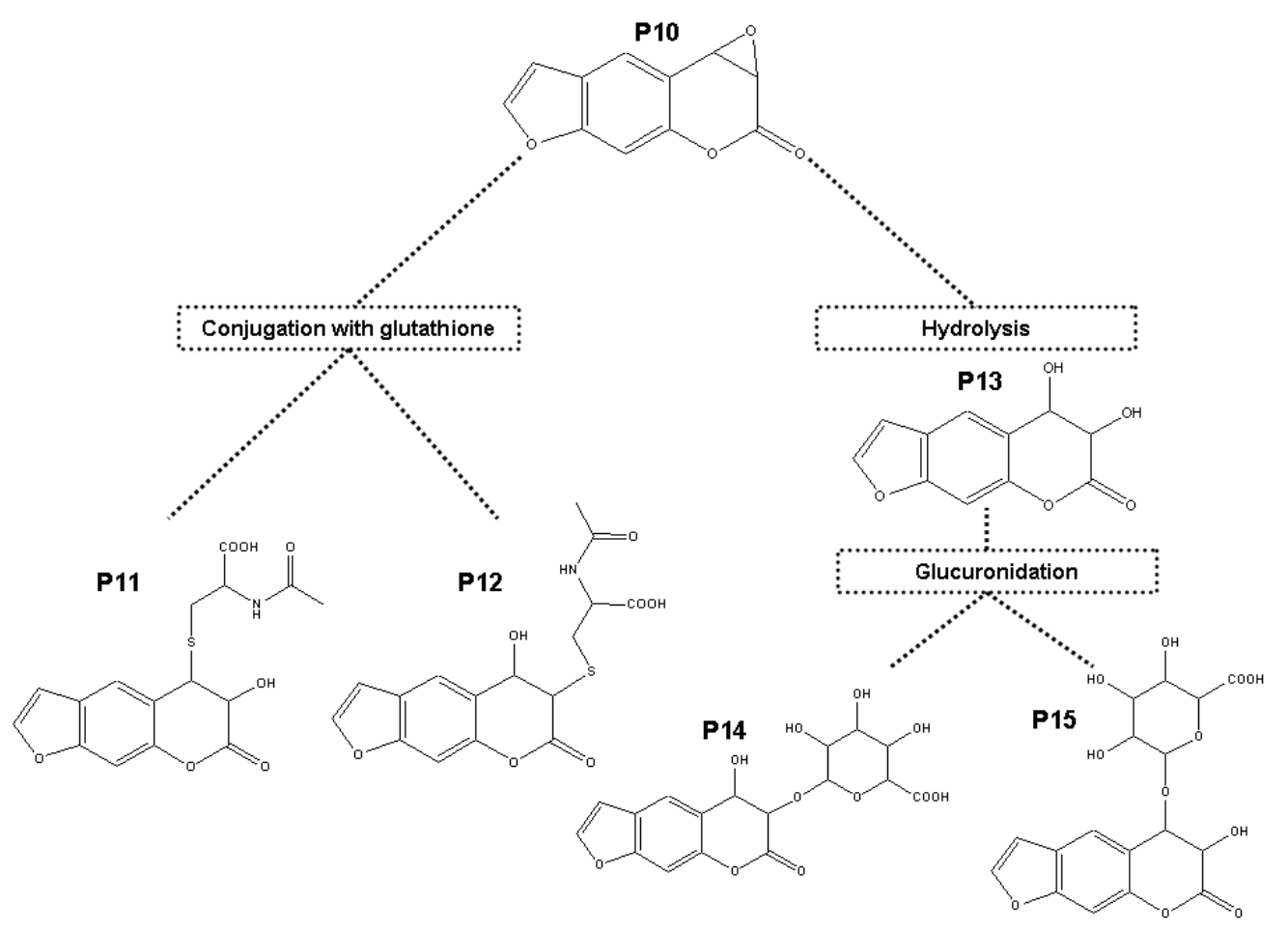

Figure 5. Metabolites and respective biotransformation reactions predicted for P10 using computational chemistry.

the same sites responsible for covalent DNA binding by which psoralens exert their phototherapeutic actions [7-9]. Therefore, at least one of these biotransformation epoxide products may be involved in the inactivation of psoralens by preventing the cross-linkage with DNA. The same can be extended to the metabolites P1 and $\mathrm{B} 1$, resulting from hydrolytic cleavage of the coumarin ring. Consequently, except for B10, which preserves the two double bonds necessary for DNA interaction, all the first-generation metabolites predicted are expected to be therapeutically inactive and, therefore, only contribute to drug elimination and, potentially, to trigger toxic responses.

According to these predictions, bergapten is expected to be more efficient than psoralen as a drug because, from the metabolic point of view, the methoxyl group in the aromatic ring can favor the biotransformation at this site and minimize the epoxidation of the double bonds. This would result in a prolonged therapeutic effect through the generation of an active metabolite (B10) that, subsequently, would be eliminated as a glucuronide conjugate (B11). All other bergapten and psoralen metabolites are expected to be inactive and will be outlined in terms of their potential to cause adverse reactions.

Regarding the in silico toxicity prediction analysis for psoralen, bergapten and their metabolites, some initial conceptual considerations are necessary. Toxicophoric groups in a molecule indicate particular substructures that are likely to be related to specific toxic effects because these chemical groups were also identified in a set of compounds (stored in molecular databases for example) that are known to trigger the same toxic responses experimentally. Whether or not the new molecule will cause the predicted toxic effect will depend on it physical chemical properties, absorption, distribution, et cetera. Nevertheless, the predicted toxic activity represents an alert, which indicates that this compound has the molecular potential to cause the toxic effect.

Psoralen and bergapten are known to cause chromosome damage and mutagenicity probably due to cross-linkage with DNA, the same mechanism observed for the therapeutic action [7-9]. Consequently, the psoralen core can be considered both a pharmacophoric and toxicophoric group (Tables 3 and 4) and modifications that drastically change the chemical 
properties and three-dimensional arrangement of the double bounds in this core are expected to reduce not only adverse effects but also the therapeutic activity.

These double bonds are also predicted to be the site of metabolization that produces epoxidated compounds, frequently associated with mutagenicity and carcinogenicity. Epoxides are electrophilic structures which, after ring opening, form a reactive ion that can act as an unspecific DNA alkylating agent [26]. Regarding the in silico toxicity prediction analysis, two psoralen ( $\mathrm{P} 4$ and $\mathrm{P} 10)$ and two bergapten metabolites (B4 and B16) exhibited the epoxide function as a toxicophoric marker while three of them were first generation metabolites (Figure 2). Therefore, this may contribute to the initial steps of carcinogenicity and mutagenicity responses associated with the administration of these drugs [10-13].

The furan ring in psoralen, bergapten and metabolites was also identified as a toxicophoric group. Furan has demonstrated to be carcinogenic in rodents and this effect can be extended to humans. The liver is the primary target for its toxicity since metabolic activation by cytochrome P450 is crucial for the carcinogenic process. The chemical mechanism is based on ring opening to generate an extremely reactive compound that alkylates nucleotides and proteins inducing an acute cytotoxic response [26-30]. According to our results, psoralen, bergapten and ten predicted metabolites each presented furan as a substructure (Tables 3 and 4), corroborating the potential of these compounds to be involved in carcinogenic episodes.

In the in silico toxicity prediction analysis, coumarin was not considered a toxic group itself but was classified as a toxicophoric marker because some of its derivatives can cause photoallergenicity. The presence of an electrondonating methyl or alkoxy group in the position 6 or 7 (Figure 6) is related to toxicity [31]. The 6- methylcoumarin, for example, has been shown to bind photochemically to human serum albumin. The mechanism of photoallergenicity seems to involve a photodimerisation reaction of the 3,4 double bond (Figure 6) enabling the formation of C4-dimers, which can acylate skin proteins [32, 33]. This mechanism depends on a photochemical reaction, where light must be absorbed by the photoallergen agent. After the initial absorption and excitation by light of an appropriate wavelength, free radicals that react with skin proteins are generated. The protein-photoallergen complex then acts as an antigen leading to the induction of an allergic response [32, 34].

Regarding psoralen, bergapten and their predicted metabolites, another toxicophoric group identified was phenyl ester, which is able to promote skin sensitization through acylation of skin proteins [35]. Resorcinol was also classified as a toxicophoric group, only present on bergapten and some of its predicted metabolites. Resorcinols are hydroxyphenols and probably react with skin proteins by a mechanism that involves the formation of phenolic radicals [36]. According to these data, the in silico prediction of metabolites containing phenyl ester, coumarin and resorcinol groups would explain the development of skin sensitization and photoallergenicty [13] associated with the administration of psoralen and bergapten.

The aim of this study was to discuss the role of psoralen and bergapten metabolites on the induction of toxicological effects, since most of the current information in this area is extrapolated from what is known about the pharmacokinetics of classical coumarins. Nevertheless, the furan substructure that distinguishes psoralens from traditional coumarins may contribute significantly to toxicity, as suggested for our results.

Although the double bonds in psoralen core are related to adverse effects, either directly or via epoxidated metabolites, they are also essential to the therapeutic action.

Table 3. Toxicophoric groups on psoralen and predicted metabolites with their corresponding toxicological effects.

\begin{tabular}{|c|c|c|}
\hline Toxicophoric Group & Molecule & Toxicity \\
\hline Psoralen & Psoralen & Chromosome damage, mutagenicity \\
\hline Furan & $\begin{array}{l}\text { Psoralen, P1, P2, P3, P10, P11, P12, } \\
\text { P13, P14, P15 }\end{array}$ & $\begin{array}{l}\text { Hepatotoxicity, carcinogenicity, } \\
\text { chromosome damage }\end{array}$ \\
\hline Epoxide & P4, P10 & $\begin{array}{l}\text { Carcinogenicity, skin sensitisation, } \\
\text { chromosome damage, mutageniciry }\end{array}$ \\
\hline Coumarin & Psoralen, P4, P5, P6, P7, P8, P9 & Photoallergenicity \\
\hline Phenyl ester & $\begin{array}{l}\text { Psoralen, P4, P5, P6, P7, P8, P9, } \\
\text { P10, P11, P12, P13, P14, P15 }\end{array}$ & Skin sensitisation \\
\hline
\end{tabular}


Table 4. Toxicophoric groups on bergapten and predicted metabolites with their corresponding toxicological effects.

\begin{tabular}{lll}
\hline Toxicophoric Group & Molecule & Toxicity \\
\hline Psoralen & Bergapten, B10, B11 & Chromosome damage, mutagenicity \\
Furan & Bergapten, B1, B2, B3, B10, B11, B12, & $\begin{array}{l}\text { Hepatotoxicity, carcinogenicity, } \\
\text { chromosome damage }\end{array}$ \\
Bhenyl esther & $\begin{array}{l}\text { Bergapten, B4, B5, B6, B7, B8, B9, B10, } \\
\text { Skin sensitisation }\end{array}$ \\
Resorcinol & B11, B16 & \\
& Bergapten, B1, B3, B4, B5, B6, B7, B8, & Skin sensitisation \\
Epoxide & B9 B10, B12, B13, B16 & Carcinogenicity, skin sensitisation, \\
& B4, B16 & chromosome damage, mutagenicity \\
Coumarin & Bergapten, B4, B5, B6, B7, B8, B9, B10, & Photoallernicity \\
\hline
\end{tabular}

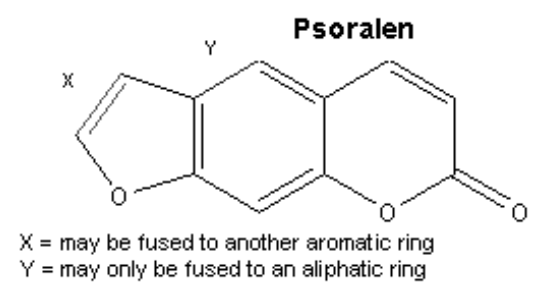

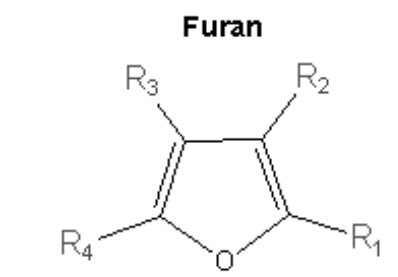

$\mathrm{R} 1-\mathrm{R} 4$ = cannot be $\mathrm{NO} 2, \mathrm{NH}, \mathrm{NC}$ or $\mathrm{COO}$ $\mathrm{R} 1-\mathrm{R} 4=$ at least one must be $\mathrm{H}$

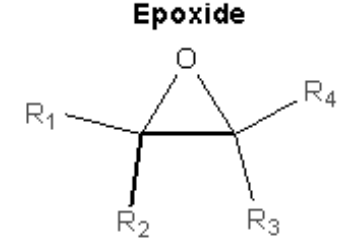

$\mathrm{R} 1-\mathrm{R} 4=$ any substituent

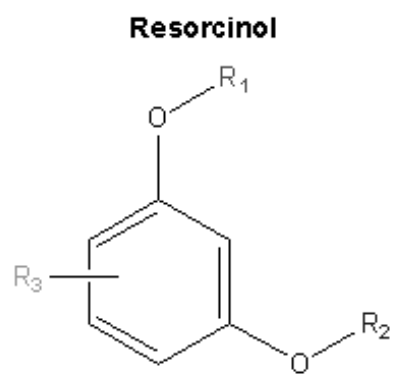

$\mathrm{R} 1=\mathrm{H}_{\mathrm{H}}$ acyl or alkyl

$\mathrm{R} 2=\mathrm{H}$ or acyl

$\mathrm{R} 3=$ any group
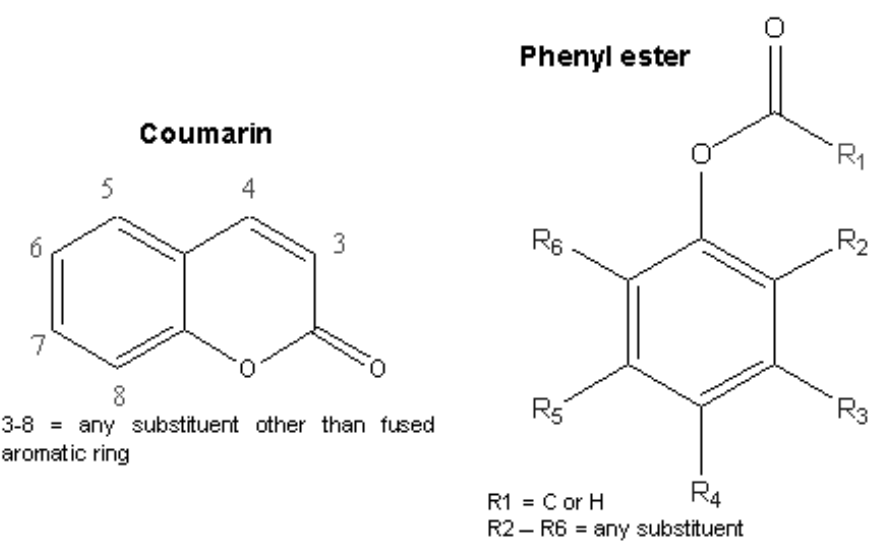

Figure 6. Chemical structures of six toxicophoric groups identified during computational chemistry analysis.

Perhaps, the design of compounds that preserve the phototerapeutic action of psoralens and have decreased levels of toxicity must focus on chemical modifications at the moieties of the psoralen core that are primarily related to the production of toxic metabolites, specifically, furan rings and coumarin substructures.

It is important to emphasize that this study is qualitative, only focusing on the potential metabolites that can be generated from psoralen and bergapten and the relationship of the chemical structure of these metabolites to classical toxicological endpoints. There is no information concerning on which metabolite would be more toxic or could be formed in greater extent.

\section{CONCLUSION}

Psoralen and bergapten are photosensibilising agents that proved to be valuable in the pharmacological treatment of vitiligo. Although the toxic effects of these drugs are well known 
and documented, there is no substantial information concerning the role of the metabolites in this process. A computational approach is particularly relevant to study this question because it may provide tools to explore and investigate the genesis of the metabolites and their toxicological effects based on literature knowledge of general metabolism reactions and structure-toxicity relationships, giving insights towards the comprehension of psoralen and bergapten pharmacokinetics and side effects. The computational analysis of psoralen, bergapten and their predicted metabolites revealed the presence of at least six toxicophoric groups related to carcinogenicity, mutagenicity, photoallergenicity, hepatotoxicity and skin sensitization. Regarding the proposed metabolites and their mechanism of action, it is possible to infer that most of them do not exhibit therapeutic effects, being relevant only for drug elimination and toxicity. We believe that the knowledge concerning the metabolic profile of psoralen and bergapten can guide the design and development of more effective and safer drugs against vitiligo.

\section{ACKNOWLEDGEMENTS}

The authors thank Fundação de Apoio à Pesquisa do Estado de São Paulo (FAPESP) for financial support.

\section{REFERENCES}

1. Sethi S, Mahajan BB, Gupta RR and Ohri A. Comparative evaluation of the therapeutic efficacy of dermabrasion, dermabrasion combined with topical 5\% 5-fluorouracil cream, and dermabrasion combined with topical placentrex gel in localized stable vitiligo. Int J Dermatol, 46(8):875-879, 2007.

2. Steiner D, Bedin V, Moraes MB, Villas RT and Steiner T. Vitiligo. An Bras Dermatol, 79(3):335-351, 2004.

3. Hernández NF, Davison GP and Sánchez GM. Vitíligo: actualización bibliográfica. Lat Am J Pharm, 27(6):938-945, 2008.

4. Dewick PM. The shikimate pathway: aromatic amino acids and phenylpropanoids, in Dewick PM (ed) Medicinal natural products: a biosynthetic approach. 2nd ed. John Wiley \& Sons: New York, NY, pp 121166, 2002.

5. Pathak MA, Daniels F and Fitzpatrick TB. The presently known distribution of furocoumarins (psoralens) in plants. J Invest Dermatol, 39:225-239, 1962.
6. Mckeon JJ. PUVA for psoriasis. Am Pharm, 9:530-532, 1981

7. Rocha MS, Viana NB and Mesquita ON. DNA-psoralen interaction: a single molecule experiment. J Chem Phys, 121(19):96799683, 2004.

8. Luftl M, Rocken M, Plewig G and Degitz K. PUVA inhibits DNA replication, but not gene transcription at nonlethal dosages. J Invest Dermatol, 111:399-405, 1998.

9. Spielmann HP, Dwyer TJ, Sastry SS, Hearst JE and Wemmer DE. DNA structural reorganization upon conversion of a psoralen furan-side monoadduct to an interstrand crosslink: implications for DNA repair. Proc Natl Acad Sci, 92:2345-2349, 1995.

10. Diawara MM, Chavez KJ, Hoyer PB, Williams DE, Dorsch J, Kulkosky $\mathrm{P}$ and Franklin MR. A novel group of ovarian toxicants, the psoralens. J Biochem Mol Toxicol, 13:195-203, 1999.

11. McKenna KE. Iatrogenic skin cancer: induction by psoralen/ultraviolet $\mathrm{A}$ and immunosuppression of organ transplant recipients. Photodermatol Photo, 20:289-296, 2004.

12. Kreimer-Erlacher H, Seidl H, Back B, Cerroni L, Kerl $\mathrm{H}$ and Wolf P. High frequency of ultraviolet mutations at the INK4a-ARF locus in squamous cell carcinomas from psoralenplus-ultraviolet-A-treated psoriasis patients. J Invest Dermatol, 120:676-682, 2003.

13. Diawara $\mathrm{MM}$ and Trumble JT. Linear furanocoumarins, in D'Mello JPF (ed) Handbook of plant and fungal toxicants. 1st ed. CRC Press: New York, NY, pp 175-188, 1997.

14. Forlot P. Toxicological and pharmacological properties of bergapten. Essenze Deriv Agrum, 70(3):147-153, 2000.

15. Pang Z, Wang B and Qi Y. Study on the pharmacokinetics of psoralen and isopsoralen by fluorometry. Chinese J Anal Chem, 29(2):146-149, 2001.

16. Lake BG. Coumarin metabolism, toxicity and carcinogenicity: relevance for human risk assessment. Food Chem Toxicol, 37:423-453, 1999.

17. Hadidi H, Zahlsen K, Idle JR and Cholerton $\mathrm{S}$. A single amino acid substitution (Leu160His) in cytochrome P450CYP2A6 causes switching from 7-hydroxylation to 3hydroxylation of coumarin. Food Chem Toxicol, 35(9):903-907, 1997.

18. Fentem JH and Fry JR. Species differences in the metabolism and hepatotoxicity of coumarins. Comp Biochem Phys C, 104(1):18, 1993.

19. Meteor Version 10.0.2 (2007). Lhasa Ltd, Leeds, UK. 
20. Derek for Windows Version 10.0.2 (2007). Lhasa Ltd, Leeds, UK.

21. Sanderson DM and Earnshaw CG. Computer prediction of possible toxic action from chemical structure; The DEREK system. Hum Exp Toxicol, 10:261-273, 1991.

22. Ridings JE, Barratt MD, Cary R, Earnshaw $\mathrm{CG}$, Eggington $\mathrm{CE}$, Ellis MK, Judson PN, Langowski JJ, Marchant CA, Payne MP, Watson WP and Yih TD. Computer prediction of possible toxic action from chemical structure: an update on the DEREK system. Toxicology, 106:267-279, 1996.

23. Greene N, Judson PN, Langowski JJ and Marchant CA. Knowledge-based expert systems for toxicity and metabolism prediction: DEREK, StAR and METEOR. SAR QSAR Environ Res, 10:299-314, 1999.

24. Testa B, Balmat A, Anthony L, and Philip J. Predicting drug metabolism: an evaluation of the expert system METEOR. Chem Biodivers, 2(7):872-85, 2005.

25. Williams DA. Drug metabolism, in Lemke TL, Williams DA, Roche VF, Zito SW (eds) Foye's principles of medicinal chemistry. $6^{\text {th }}$ ed. Lippincott Williams \& Wilkins: New York, NY, pp 253-326, 2008.

26. Hine C, Rowe VK, White ER, Darmer KI and Youngblood GT. Epoxy compounds, in Clayton GD and Clayton FE (eds) Patty's Industrial Hygiene and Toxicology. 3rd ed. John Wiley: New York, NY, pp 2141-2257, 1981.

27. Peterson LA, Naruko KC and Predecki D. A reactive metabolite of furan, cis-2-butene-1,4dial, is mutagenic in the Ames assay. Chem Res Toxicol, 13:531-34, 2000.

28. Chen LJ, Hecht SS and Peterson LA. Identification of cis-2-butene-1,4-dial as a microsomal metabolite of furan. Chem Res Toxicol, 8(7):903-906, 1995.

29. Burka LT, Washburn KD and Irwin RD. Disposition of [14C]furan in the male F344 rat. J Toxicol Environ Health, 34(2):245-257, 1991.

30. Byrns MC, Predecki DP and Peterson LA. Characterization of nucleoside adducts of cis2-butene-1,4-dial, a reactive metabolite of furan. Chem Res Toxicol, 15(3):373-79, 2002.

31. Kaidbey $\mathrm{KH}$ and Kligman AM. Photosensitization by coumarin derivatives. Arch Dermatol, 117:258-263, 1981.

32. Barratt MD and Brown KR. Photochemical binding of photoallergens to human serum albumin: a simple in vitro method for screening potential photoallergens. Toxicol Lett, 24:1-6, 1985.

33. Kato S, Seki T, Katsumura Y, Kobayashi T, Komatsu K and Fukushima S. Mechanism for 6-methylcoumarin photoallergenicity. Toxicol Appl Pharmacol, 81(2):295-301, 1985.

34. Delahanty JN, Evans JC, Rowlands CC and Barratt MD. An electron spin resonance study of the free radicals formed on ultraviolet irradiation of the photoallergens fentichlor and bithionol. J Chem Soc Faraday Trans I, 83:135-139, 1987.

35. Barratt MD, Basketter DA and Roberts DW. Skin sensitization structure-activity relationships for phenyl benzoates. Toxicol in Vitro, 8:823-826, 1994.

36. Barratt M.D and Basketter DA. Possible origin of the skin sensitization potential of isoeugenol and related compounds: (I) preliminary studies of potential reaction mechanisms. Contact Derm, 27:98-104, 1992. 